\title{
EDUCAÇÃO EM SAÚDE: POR QUEM E PARA QUEM? A VISÃO DE ESTUDANTES DE GRADUAÇÃO EM ENFERMAGEM
}

\author{
Juliana Silveira Colomé1, Dora Lúcia Leidens Corrêa de Oliveira²
}

\footnotetext{
${ }^{1}$ Doutoranda em Enfermagem da Universidade Federal do Rio Grande. Docente do Curso de Enfermagem do Centro Universitário Franciscano. Rio Grande do Sul, Brasil. E-mail: julianacolome@yahoo.com.br

${ }^{2}$ Doutora em Enfermagem. Docente da Escola de Enfermagem da Universidade Federal do Rio Grande do Sul. Rio Grande do Sul, Brasil. E-mail: dora@enf.ufrgs.br
}

RESUMO: Estudo exploratório-descritivo de abordagem qualitativa. Teve como objetivo analisar as concepções de estudantes de enfermagem acerca da educação em saúde e quais são os seus sujeitos e agentes. Os campos de desenvolvimento do estudo foram os Cursos de Graduação em Enfermagem da Universidade Federal de Santa Maria e da Universidade Federal do Rio Grande do Sul. Participaram da pesquisa os estudantes do último semestre dos respectivos cursos. A coleta de dados foi realizada por meio de entrevista semiestruturada e os dados foram submetidos à análise de conteúdo temática. Os resultados sugerem que o trabalho educativo em saúde é permeado por concepções que mesclam pressupostos tradicionais e pressupostos ampliados de educação em saúde, embora os primeiros sejam prevalentes. Percebe-se o predomínio de concepções com foco no saber biomédico, traduzido por experiências curriculares pautadas em ações preventivistas, com ênfase em saberes técnico-científicos e em mudanças individuais de comportamento. DESCRITORES: Educação em saúde. Promoção da saúde. Educação em enfermagem.

\section{HEALTH EDUCATION: BY WHOM AND FOR WHOM? THE VISION OF NURSING UNDERGRADUATES STUDENTS}

\begin{abstract}
The objective of this descriptive, exploratory study with a qualitative approach was to analyze the conceptions of Brazilian nursing undergraduates about health education and its subjects and agents. It was developed in the Undergraduate Nursing Courses of the Federal University of Santa Maria and the Federal University of Rio Grande do Sul, Brazil. The research participants were enrolled in their last semester before graduation. Data was collected using a semi-structured interview and submitted to thematic content analysis. The results suggest that health education work is permeated by conceptions that blend traditional and enlarged health education assumptions, although the former are more prevalent. Through this study, one perceives the dominance of conceptions which focus on biomedical knowledge translated through curricular experiences guided by preventive actions that emphasize technical and scientific knowledge and individual behavior changes.
\end{abstract}

DESCRIPTORS: Health education. Health promotion. Education, nursing.

\section{EDUCACION EN LA SALUD: POR QUIEN Y PARA QUIEN? LA VISION DE LOS ESTUDIANTES DE GRADUACIÓN EN ENFERMERIA}

\begin{abstract}
RESUMEN: Estudio exploratório, descriptivo, con enfoque cualitativo. Tuvo como objetivo analizar los conceptos de los estudiantes de enfermería sobre la educación para la salud y cuáles son sus sujetos y agentes. Las áreas de desarrollo del estudio fueran los Cursos de Enfermería de la Universidad de Santa Maria y de la Universidad Federal do Rio Grande do Sul. Participaran de la investigación los estudiantes del último semestre de los respectivos cursos. La colecta de datos fue realizada mediante entrevista semi-estructurada y los datos fueran sometidos al análisis del contenido temático. Los resultados sugieren que el trabajo educativo en la salud es infiltrado por concepciones que mesclan presupuestos tradicionales y hipótesis ampliadas de educación en salud, aunque los primeros sean más frecuentes. Se puede observar el predominio de los conceptos con un enfoque en el conocimiento biomédico, traducido por guiar experiencias curriculares en los programas de prevención, con énfasis en los conocimientos técnicos y científicos y los cambios en el comportamiento individual.
\end{abstract}

DESCRIPTORES: Educación para la salud. Ascensión de la salud. Educación en enfermería. 


\section{INTRODUÇÃO}

A educação em saúde constitui-se tanto como um espaço importante de construção e veiculação de conhecimentos e práticas relacionados aos modos como cada cultura concebe o viver de forma saudável, quanto como uma instância de produção de sujeitos e identidades sociais. ${ }^{1}$ No campo das práticas de saúde, existe uma diversidade de modelos de educação em saúde e, considerando o que estas abordagens têm em comum, é possível agrupá-las em duas vertentes principais: o modelo tradicional ou preventivo e o modelo radical. ${ }^{2}$

O modelo tradicional de educação nem saúde apóia-se num entendimento de saúde como ausência de doença e na proposição de estratégias educativas orientadas por pressupostos biomédicos. Nesse contexto, as ações de educação em saúde têm caráter persuasivo, pois buscam prescrever determinados comportamentos considerados ideais para a prevenção ou minimização de agravos à saúde. A abordagem tradicional de educação em saúde caracteriza-se, ainda, pela identificação dos profissionais da saúde como legítimos executores ou agentes de ações educativas em saúde. ${ }^{3}$

Segundo essa visão, como os profissionais da saúde são aqueles que conhecem as escolhas mais saudáveis, estão autorizados a convencer os indivíduos a adotar um estilo de vida compatível com estas escolhas. A possibilidade de alguém optar por atitudes consideradas não saudáveis, após ter participado de algum tipo de atividade educativa em saúde, é interpretada pelos profissionais como uma falha na eficácia destas ações. Além disso, na educação em saúde tradicional, as escolhas consideradas saudáveis pelos profissionais da saúde são propostas como as únicas opções possíveis e disponíveis aos indivíduos. ${ }^{4-5}$

Em contrapartida, a necessidade de uma aproximação com referenciais ampliados, tem resultado em ações educativas em saúde que levam em consideração a complexidade do fenômeno saúde e que consideram uma perspectiva ampliada de educação. Essa abordagem educativa em saúde, denominada radical, ${ }^{2}$ busca o fortalecimento da consciência crítica das pessoas, transferindo o foco das ações educativas tradicionalmente centradas no indivíduo para um investimento no potencial dos grupos sociais.

A educação em saúde radical apóia-se nos pressupostos da promoção da saúde, uma proposta que buscou renovar e transformar as práticas educativas no campo da saúde, predominante- mente centradas na prevenção de doenças. Para a promoção da saúde e, portanto, para a abordagem radical da educação em saúde, somente a prevenção de doenças não poderá ser considerada como um objetivo suficiente, já que perante qualquer nível de saúde, sempre haverá algo a ser feito para promover condições de vida mais satisfatórias.

O principal subsídio desta mudança de perspectivas reside no próprio conceito de saúde, a qual é entendida num sentido positivo e multidimensional. Os modelos de intervenção educativos propostos são essencialmente participativos, sendo oferecidos à população com a finalidade de abordar uma vasta rede de temas relacionados à saúde. Na perspectiva radical, a ampliação do escopo de saberes e práticas orientadores da educação para a promoção da saúde, possibilita uma abertura ao trabalho de agentes que não sejam exclusivamente os profissionais da saúde. ${ }^{3}$

A compreensão de educação em saúde a partir de um conceito ampliado de saúde sugere o reconhecimento da insuficiência do modelo biológico, da tecnologia médica e do foco exclusivo no risco individual para responder aos processos de saúde e não-saúde. ${ }^{6}$ Diferente da compreensão de educação em saúde tradicional, indicada anteriormente, esta concepção orienta-se pela ideia de que as ações educativas em saúde desenvolvidas na perspectiva radical objetivam promover a autonomia dos sujeitos a partir de suas próprias escolhas, de forma desvinculada da regulação e supervisão dos profissionais da saúde. ${ }^{7-8}$

Nessa perspectiva, por mais que ambos modelos de educação em saúde apresentem pontos problemáticos, acredita-se que a abordagem radical seja a mais coerente com os pressupostos da promoção da saúde, pois estimula os indivíduos a assumir um maior controle sobre suas vidas por meio de atitudes críticas relacionadas não somente ao individual, mas também à coletividade.

Embora não exista legislação que indique como um imperativo o desenvolvimento de ações educativas em saúde por enfermeiros, estes têm sido alguns dos seus principais agentes. Em função do conhecimento adquirido no meio acadêmico, que os instrumentaliza no conhecimento dos processos de adoecimento humano e, consequentemente, nas diversas e abrangentes formas de preveni-los, os enfermeiros são aqueles que frequentemente assumem o desafio de educar para a saúde. ${ }^{9}$

Nessa direção, o enfermeiro tem se constituído como um importante agente de ações 
educativas em saúde, sobretudo, nos espaços institucionalizados de saúde. Pelo conhecimento amplo e contextualizado, específico de sua formação, o enfermeiro pode ser considerado um profissional qualificado para propor e redefinir as práticas de saúde, por meio de ações educativas voltadas tanto para a organização do processo de trabalho em saúde, quanto para o fomento de práticas sociais empreendedoras, voltadas para a promoção e proteção da saúde dos indivíduos, famílias e comunidades. ${ }^{10}$

Gradativamente, é preciso ampliar as possibilidades teórico-reflexivas, por meio de abordagens problematizadoras que instiguem práticas inovadoras e transformadoras, capazes de abrir caminhos para a produção social e organizada dos processos de trabalho em saúde. Diante destas reflexões e considerando a importância da dimensão educativa na prática cotidiana da organização do trabalho em enfermagem, há a necessidade do desenvolvimento de uma análise crítica não somente desta prática, mas também da formação do enfermeiro influenciada por esta.

Com base no exposto, este artigo objetiva analisar as concepções de estudantes de enfermagem acerca da educação em saúde e quais são seus sujeitos e agentes.

\section{METODOLOGIA}

O presente artigo tem origem na Dissertação de Mestrado intitulada "A formação de educadores em saúde na graduação em enfermagem: concepções dos graduandos", ${ }^{11}$ desenvolvida no Programa de Pós-graduação em Enfermagem da Universidade Federal do Rio Grande do Sul (UFRGS). O estudo foi de cunho qualitativo e desenvolvido numa abordagem exploratório-descritiva. Os campos de estudo foram os Cursos de Graduação em Enfermagem da Universidade Federal de Santa Maria (UFSM) e da UFRGS. A escolha dessas duas Instituições de Ensino Superior (IESs) justifica-se em função de que as mesmas representam importantes pólos formativos para a educação em enfermagem, tanto do Estado do Rio Grande do Sul, como de outras regiões do país. Ainda, a realização da pesquisa nesses dois cenários trouxe a possibilidade de ampliar o olhar sobre o objeto de estudo, analisando realidades potencialmente diferentes.

Foi utilizada uma amostragem intencional, na qual participaram da pesquisa os estudantes do último semestre dos referidos cursos, os quais se encontravam na fase do Estágio Supervisionado em Enfermagem. Em cada um dos cursos foram sorteados 10 estudantes para participarem da pesquisa, perfazendo um total de 20 participantes. A definição da amostra do estudo atentou para que cada um dos cursos tivesse o mesmo número de participantes, de forma a não haver privilégio de uma IES em detrimento da outra. Salienta-se que todos os estudantes selecionados aceitaram participar da pesquisa. Como não emergiram divergências significativas no conteúdo das falas dos estudantes das duas universidades que participaram do estudo, optou-se por analisar estas falas sem relacioná-las ao curso a que estes estudantes pertenciam.

No intuito de preservar a identidade dos sujeitos foi adotada a denominação "Grad." (forma abreviada de graduando), seguida de uma numeração de 1 a 20, por exemplo: Grad. 1, Grad. 2. Destaca-se ainda, que os participantes identificados no intervalo Grad.1 ao Grad. 10 correspondem aos estudantes da UFSM, ao passo que os participantes identificados como Grad. 11 até Grad. 20 correspondem aos estudantes da UFRGS.

A coleta de dados foi realizada mediante a utilização de um roteiro de entrevista semiestruturado, no qual os participantes responderam os seguintes questionamentos: para você, quem são as pessoas que estão preparadas para realizar ações educativas em saúde? Por quê? Durante o curso, quem foram os principais sujeitos das ações educativas em saúde realizadas pelos alunos? Em que locais e/ou circunstâncias estas ações aconteceram? Que estratégias foram utilizadas para a realização destas ações?

Os dados foram submetidos à análise de conteúdo temática, a qual permite contemplar os dados empíricos em suas peculiaridades, em seu contexto social e histórico. ${ }^{12}$ A análise dos dados foi operacionalizada por meio dos seguintes passos: pré-análise, exploração do material, tratamento dos resultados obtidos e interpretação. Na fase de pré-análise foram realizadas as transcrições das falas, a releitura e a organização do material, obtendo-se uma visão geral dos dados coletados. Na etapa de exploração do material, os dados coletados foram discutidos juntamente com o referencial teórico, seus pressupostos e hipóteses. O tratamento dos resultados obtidos e sua interpretação constituíram a articulação entre o material empírico e o referencial teórico, a fim de realizar uma reflexão capaz de ultrapassar a simples descrição dos dados e estabelecer relações 
que permitissem novas interpretações. O processo analítico deu origem a duas categorias principais: Educação em saúde: para quem? e Educação em saúde: por quem?

O projeto foi aprovado pelo Comitê de Ética em Pesquisa da UFRGS, parecer $\mathrm{n}^{\circ} 2006579$, e os participantes da pesquisa, após serem esclarecidos sobre o processo investigativo e seus objetivos, aceitaram voluntariamente dela participar, assinando o Termo de Consentimento Livre e Esclarecido.

\section{RESULTADOS E DISCUSSÃO}

O processo de análise de dados permitiu que os mesmos fossem agrupados em duas categorias principais. A primeira delas, intitulada "Educação em saúde: para quem?", discute quais seriam os sujeitos das ações educativas em saúde, ou seja, para quais pessoas tais práticas deveriam ser direcionadas. Por sua vez, a segunda categoria analítica, "Educação em saúde: por quem?", aborda as concepções dos estudantes de enfermagem em relação aos agentes da educação em saúde, aquelas pessoas que, no seu entendimento, estão autorizadas a desenvolvê-la.

\section{Educação em saúde: para quem?}

As entrevistas com os estudantes permitiram observar que, nas ações de educação em saúde vivenciadas na graduação em enfermagem, os sujeitos das ações educativas eram representantes de grupos específicos, em sua maioria, indivíduos com doenças crônico-degenerativas, especialmente diabetes e hipertensão arterial. Os dados indicam que a definição de quem deve ser sujeito das ações educativas em saúde é embasada no pressuposto de que pessoas que compartilham o mesmo processo crônico de adoecimento seguem estilos de vida muito semelhantes, com comportamentos e atitudes pessoais diretamente implicados na origem da doença. ${ }^{2}$

Após ser definido o problema da doença crônica, buscam-se soluções que investem em mudanças individuais de comportamento, em detrimento a intervenções mais amplas, que considerem a influência do meio nas escolhas e comportamentos individuais. Pode-se inferir que existe uma prática educativa em saúde constante na formação do enfermeiro, mas de uma forma restrita, intervencionista, com ênfase na mudança de hábitos e principalmente abordada nos grupos de saúde, os quais poderiam ser intitulados como grupos de doença ${ }^{13}$ Tinha sempre grupos de pessoas com diabetes, hipertensão, onde então os grupos eram direcionados para estas patologias (Grad.4). Teve grupo de fibrose cística, mulheres no climatério, hipertensos, asmáticos, diabéticos, grupos que a gente realizou e também proporcionou educação para a saúde (Grad.12).

Segundo os entrevistados, também pertenciam ao conjunto de indivíduos cujas necessidades deveriam ser atendidas pela via da educação em saúde, pessoas carentes de conhecimento ou com baixa escolaridade e renda, fatores que os legitimariam na posição de sujeitos prioritários dos investimentos da enfermagem no campo educativo em saúde. Neste ensino de como se educa as pessoas para que tenham ou recuperem sua saúde, percebe-se uma problemática ligação entre necessidade de educação em saúde e baixa renda.

A ideia de que a pobreza, a escolaridade formal e a renda são parâmetros para avaliar a necessidade de envolvimento da educação em saúde, revela uma tendência das classes mais favorecidas em secundarizar os saberes das classes populares. ${ }^{14}$ Nas falas dos estudantes percebe-se esta compreensão simplista de que há uma 'natural' carência de saber entre pessoas pobres, a qual é justificada por suas precárias condições de vida: a educação em saúde foi sempre para as pessoas de baixa renda e pouca escolaridade, pessoas basicamente leigas, pessoas que tu sabe que estão desinformadas, vivendo em condições precárias (Grad.5).

Ações educativas em saúde, desenvolvidas nessa perspectiva, reproduzem uma forma de assistência fundamentada no repasse de informações e na ênfase no saber técnico, não contribuindo para a troca de conhecimentos. Assim, pode-se dizer que, mesmo que os estudantes considerem a educação em saúde como um elemento essencial no cuidado, tais práticas vêm sendo realizadas de forma vertical, com funções delimitadas de quem é o educador e quem é o educando, ou seja, quem tem o poder de ensinar e quem deve aprender. ${ }^{13}$

Embora a ênfase dos depoimentos tenha sido em uma modelagem de educação em saúde que reitera concepções de saúde como ausência de doença, e de educação como transmissão vertical de informações, a análise indica, no posicionamento de alguns graduandos, 'modos de ver' que se contrapõem a esta forma tradicional de fazer educação.

Sugerindo foco na dimensão emancipatória da educação em saúde, alguns graduandos expressaram em suas falas que os indivíduos envolvidos nas ações educativas exercem um papel 
protagonista no contexto destas ações. A referência a estes protagonistas indica uma compreensão de que os sujeitos da educação em saúde possuem saberes e experiências que devem ser valorizados nos processos educativos, sinalizando para uma possível evolução do ideário que tradicionalmente sustenta a formação de enfermeiros.

Embora alguns depoimentos insinuem esta evolução, provavelmente ainda são poucas as experiências acadêmicas que promovem aprendizados que extrapolam concepções mais tradicionais do que seja educar para promover saúde. Nestas 'exceções educativas', os sujeitos das ações de educação em saúde foram chamados para delas participarem em função de ocuparem uma posição estratégica nas suas comunidades, como é o caso de líderes comunitários. Sua inclusão no perfil dos possíveis sujeitos das ações educativas foi relacionada ao seu status social, ou seja, por serem considerados personagens importantes na discussão de questões de interesse para as comunidades que representam.

A indicação destes líderes como sujeitos das ações de educação em saúde sugere a possibilidade de uma relativa ampliação das experiências educativas em saúde oferecidas nos cursos de graduação, alargando o escopo de atuação dos enfermeiros: [...] trabalhei com grupos de pastoral da saúde e nesses grupos nós procuramos embasar todas as ações de educação em saúde em função das necessidades da comunidade. Inicialmente procuramos conhecer o grupo, suas percepções e o que achariam necessário, o que achariam importante para a comunidade (Grad.6).

Depoimentos como este e os argumentos que a eles podem ser articulados, sinalizam para um investimento em novas maneiras de perceber os sujeitos das ações educativas em saúde. Parece estar implícita aí uma tendência em conceber tais ações como projetos coletivos, definidos em função de interesses comuns a ambos, agentes e sujeitos da educação pretendida. Pensar na promoção da saúde pela via da educação, contando como sujeitos das ações educativas pessoas que conheçam a realidade e as necessidades de determinados grupos sociais, supõe propor uma educação em saúde para a emancipação, a qual se fundamenta no diálogo e na interação entre as pessoas. Neste tipo de contexto educativo, por mais que a argumentação dos profissionais provenha de sua capacitação técnica, não se exclui nem o conhecimento, nem os aspectos humanos colocados pelos sujeitos, buscando de forma conjunta encontrar a melhor forma de enfrentar as situações. ${ }^{15}$
Outro depoimento sugeriu como sujeitos de ações de educação em saúde, indivíduos submetidos a condições precárias de vida. Por exemplo, seguindo o viés da participação e da emancipação, o depoimento que segue considerou estes indivíduos como partícipes na busca, em conjunto com os agentes educativos, de propostas de ação que transcendessem aquelas orientadas para a clínica, investindo em novos caminhos para a busca da qualidade de vida e indicando uma concepção de saúde que extrapola os limites da ausência de doença:

[... ] este grupo tinha várias precariedades, várias condições que os dificultavam, mas o objetivo maior foi o alcance da qualidade de vida das crianças e de suas famílias e ligando com o serviço local, procurar não atendê-los de uma forma tão clínica, mas prestar um cuidado de enfermagem diferenciado. Acho que não transmiti de uma forma vertical os meus conhecimentos, porque pelos relatos a gente compartilhou bastante coisa (Grad.19).

Esta abordagem parece condizente com o modelo radical de educação em saúde, o qual propõe a superação da ênfase transmissiva da educação com vistas a um reposicionamento do educador e do educando numa relação mais horizontal. Nesta perspectiva, a definição das necessidades de educação e dos temas a serem trabalhados nos espaços educativos não seria mais uma responsabilidade dos profissionais da saúde, mas resultado de um processo coletivo de problematização da realidade vivida realizado com vistas à transformação desta realidade.

O potencial transformador deste tipo de ação é reafirmado na literatura no argumento de que, quanto mais as massas populares desvelam a realidade objetiva e desafiadora sobre a qual devem incidir sua ação transformadora, tanto mais se inserem nela criticamente, sendo que a liderança e as massas, cointencionadas, encontram-se em uma tarefa em que ambas são sujeitos no ato, não somente de desvelar a realidade, mas criticamente de recriar esta realidade. Ao alcançarem na reflexão e na ação em comum este saber da realidade, estes parceiros se descobrem como seus refazedores permanentes e se direcionam a novas ações e soluções. ${ }^{16}$

Desse modo, há que se repensar o ensino de enfermagem, a fim de que esteja pautado em processos formativos com identificação nas necessidades de saúde da população, bem como na concepção social de cuidado de enfermagem. A articulação entre teoria e prática no ensino da enfermagem, por sua vez, pressupõe ações peda- 
gógicas que valorizem a inserção dos usuários nos espaços de produção do cuidado, aí incluídos os relativos à educação em saúde. Nessa perspectiva, destaca-se a importância de que a formação em enfermagem seja centrada numa contínua aproximação do ensino com o mundo do trabalho. ${ }^{17}$

O necessário reposicionamento do educador nestes cenários implicaria a aceitação de que profissionais de saúde não são os únicos detentores de saberes sobre saúde. Neste cenário seria mais apropriado reconhecer os saberes dos indivíduos, para os quais se encontram direcionados as ações educativas em saúde, como igualmente relevantes, e ocupar um papel de mediador de processos de compartilhamento e não de imposição de saberes, processos estes constituídos em espaços educativos de caráter dialógico.

\section{Educação em saúde: por quem?}

No que se refere aos agentes da educação em saúde, destaca-se que os enfermeiros foram referidos como possuidores dos atributos necessários para realizar educação em saúde. Além dos enfermeiros, outros representantes das profissões da saúde foram considerados como agentes autorizados a planejar, coordenar e desenvolver ações educativas em saúde: [...] os profissionais da área da saúde, por ter um conhecimento mais aprofundado, então a enfermagem, a fisioterapia, podem fazer educação em saúde. O profissional da saúde por estudar mais sobre as patologias tem a saúde mais presente nas matérias do currículo (Grad.3); A educação em saúde se refere mais à prevenção de doenças, então quem tem mais conhecimento sobre essa área seriam os profissionais que estão na área da saúde, os enfermeiros principalmente (Grad.15).

Esses dados sugerem que a visão de saúde como ausência de doença e do conhecimento em saúde como restrito ao campo biomédico, resulta na óbvia indicação de profissionais reconhecidos como detentores deste conhecimento como preferenciais e legítimos agentes de educação em saúde. Esta percepção está, provavelmente, relacionada à convicção de que educar para promover a saúde se constitui em um investimento limitado à esfera de domínio dos profissionais da saúde, em função da necessidade de priorizar-se a sabedoria técnico-científica. ${ }^{1}$

Evidencia-se, na perspectiva dos estudantes, que o saber profissional definido pela ciência biomédica, figura como um dos instrumentos essenciais para a efetividade de ações educativas em saúde. O posicionamento dos estudantes de enfermagem sugere que, como referido na literatura, no cotidiano das ações educativas, o profissional de saúde por se considerar técnico e especialista, ainda acredita possuir, sob a forma de monopólio, o conhecimento verdadeiro sobre os temas implicados com saúde. ${ }^{18}$

Ao contrário daqueles que possuem uma visão mais tradicional da educação em saúde, outros estudantes consideraram que 'fazer educação em saúde' não é prerrogativa apenas dos profissionais da saúde e que essa prática se configura como um campo de parceria entre profissionais da saúde, profissionais de outras áreas e membros da comunidade.

A ênfase dos graduandos na atuação conjunta de profissionais de diversas áreas revela uma lógica interdisciplinar na compreensão do fenômeno saúde. Reconhecer profissionais das mais variadas disciplinas como potenciais educadores em saúde é uma forma de relativizar o poder de determinadas profissões para promover saúde, em detrimento de outras: [...] eu acho que não precisaria o enfermeiro ter que atuar sozinho na educação em saúde, poderia trabalhar mais com o assistente social, com o nutricionista, com o psicólogo, porque a gente precisa de outros profissionais e a interdisciplinaridade é essencial para trabalhar educação em saúde (Grad.1).

Tomando como parâmetro a tradicional compreensão de que os profissionais da saúde são os agentes com maior legitimidade para desenvolver ações de educação em saúde, percebe-se como um avanço o fato de alguns graduandos reconhecerem a necessidade de práticas interdisciplinares neste campo. Apesar de se considerar a necessidade de projetos educativos interdisciplinares no campo da saúde, é preciso pontuar que a lógica interdisciplinar que permeia as falas dos estudantes abrange, somente, profissionais das áreas da saúde e da educação, revelando um entendimento ainda restrito de interdisciplinaridade, dado o amplo leque de disciplinas implicadas com o fenômeno saúde. ${ }^{19}$

Os estudantes também referiram a possibilidade de que membros da comunidade atuem como educadores em saúde. Esta participação daqueles que se deslocam da qualidade de sujeitos para assumir o papel de agentes, parece implicar, mais uma vez, num significativo progresso no contexto da educação em saúde, uma vez que os agentes até então lembrados pelos estudantes deveriam possuir um diploma de graduação ou terem sido preparados por profissionais graduados, como no caso dos agentes comunitários de 
saúde. O depoimento que segue exemplifica o entendimento dos alunos:

\section{[...] acho que até quem não fez faculdade pode ser} educador em saúde, pois uma pessoa da comunidade tem informações que não deixam de ser educação em saúde. A gente vê que tem pessoas que tem conhecimento, de repente eles não vão saber passar da maneira científica, mas vão saber conversar com os outros (Grad.11).

O envolvimento de membros da comunidade como agentes de educação em saúde remete também a uma nova relação entre usuários e profissionais, onde ambos são posicionados como protagonistas destas ações. As estratégias de educação em saúde, inicialmente restritas ao campo de ação de profissionais da saúde, necessitam ser também uma responsabilidade da população organizada com interesses em saúde e de atores de outros setores sociais não diretamente envolvidos com a área da saúde. ${ }^{3}$ Neste contexto, destaca-se o importante papel dos educadores em saúde, sejam profissionais ou membros da comunidade, em relação ao compromisso com a produção de saúde e com a produção de sujeitos autônomos, reflexivos e socialmente solidários. ${ }^{20}$

$\mathrm{Na}$ análise dos sujeitos e agentes da educação em saúde apontados pelos participantes da pesquisa, destaca-se que tanto nos processos formativos destes estudantes, como nas práticas educativas que são desenvolvidas nos campos de prática, existe uma coexistência de pressupostos tradicionais e de pressupostos ampliados de educação em saúde. O foco na formação de um profissional comprometido em transcender a tradicional lógica informativo-preventivista e subsidiado essencialmente por um referencial emancipatório-participativo, já deveria ser predominante no interior dos processos formativos.

\section{CONSIDERAÇÕES FINAIS}

A análise das concepções dos graduandos de enfermagem acerca dos sujeitos e dos agentes das ações educativas em saúde revelou que este processo, apesar das mudanças paradigmáticas que vem permeando o campo da educação em saúde, ainda encontra-se fortemente subsidiado por pressupostos biomédicos, traduzidos em ações educativas em saúde orientadas para práticas transmissivas e finalidades preventivistas. Nos depoimentos dos alunos, ficou clara a ênfase da formação para o trabalho educativo em saúde nos conhecimentos científicos sobre doenças, prevenção e intervenções terapêuticas, em detrimento de outros conhecimentos igualmente implicados com o fenômeno saúde.

O entendimento de saúde e de não-saúde como resultantes de comportamentos individuais equivocados originados, principalmente, em lacunas de conhecimento parece justificar o investimento na formação de enfermeiros-educadores capazes de transmitir informações sobre saúde, julgadas relevantes e concebidas desde um ponto de vista biomédico. Neste contexto, destaca-se a abordagem das doenças crônicas e das respectivas ações de autocuidado, temáticas reconhecidas pelos graduandos como elementos legítimos do arcabouço de saberes que configuram 'saúde' como um conteúdo a ser ensinado.

Por outro lado, ainda que, com menos frequência, alguns depoimentos trouxeram elementos de uma noção ampliada de educação em saúde, a qual extrapola atividades centradas na doença e busca enfatizar os múltiplos determinantes da saúde. Na análise destes depoimentos foi possível identificar alguns dos pressupostos da promoção da saúde, o que sugere uma tendência de superação da lógica preventivista, de transcendência do objetivo da mudança de comportamento individual para investimentos em transformações das condições estruturais de vida.

Estes dados sugerem que, o processo formativo em estudo é permeado por concepções que mesclam pressupostos tradicionais e pressupostos ampliados de educação em saúde, embora os primeiros sejam prevalentes. A dificuldade de uma implementação prática dos referenciais que sustentam a promoção da saúde, é justificada pelo forte predomínio do saber biomédico na formação de enfermeiros, que se traduz principalmente em experiências curriculares que objetivam instrumentalizar os alunos para ações educativas em saúde preventivistas, com ênfase em mudanças individuais de comportamento e pautadas em saberes técnico-científicos.

Os depoimentos analisados sinalizam para a necessidade de repensar a formação do enfermeiro para atuação no campo da educação em saúde. De acordo com o entendimento dos estudantes, muitas vezes, estas ações estão direcionadas para um processo que não vem contemplando a visão de mundo dos sujeitos, limitando-se, muitas vezes, ao autoritarismo do profissional, numa atuação que não estimula o desenvolvimento da consciência crítica dos envolvidos, não divide responsabilidades e ainda não reconhece os sujeitos das ações educativas como atores deste processo. 
Destaca-se neste contexto, a responsabilidade das universidades e dos centros formadores com a preparação de profissionais da saúde comprometidos com as necessidades sociais, com o fortalecimento do SUS e com projetos educativos contextualizados à realidade. Para que os processos de formação possam preparar os profissionais da saúde para dar conta destas necessidades, é necessário investir na formação de profissionais críticos e capazes de refletir sobre sua realidade social, política, cultural e, portanto, instrumentalizados para viabilizar rupturas em práticas instituídas.

\section{REFERÊNCIAS}

1. Meyer DE. "Você aprende. A gente ensina?": interrogando relações entre educação e saúde desde a perspectiva da vulnerabilidade. Cad Saúde Pública 2006 Jun; 22(6):1335-42.

2. Oliveira DLLC. A "nova" saúde pública e a promoção da saúde via educação: entre a tradição e a inovação. Rev Latino-am Enferm. 2005 Mai-Jun; 13(3):423-31.

3. Buss PM. Uma introdução ao conceito de promoção da saúde. In: Czeresnia D, Freitas CM, organizadores. Promoção da saúde: conceitos, reflexões, tendências. Rio de Janeiro: Fiocruz, 2003. p.15-38.

4. Gastaldo D. É a educação em saúde saudável? Repensando a educação em saúde através do conceito de bio-poder. Rev Educação Realidade. 1997 Jan-Jun; 22(1):147-68.

5. Souza AC, Colomé ICS, Costa LED, Oliveira DLLC. A educação em saúde com grupos na comunidade: uma estratégia facilitadora da promoção da saúde. Rev Gaúcha Enferm. 2005 Ago; 26(2):147-53.

6. Marcondes WB. A convergência de referências na promoção da saúde. Saúde Soc. 2004 Mai-Ago 13(2):5-13.

7. Chiesa AM, Veríssimo MDLO. A educação em saúde na prática do PSF. Manual de enfermagem [online]. Brasília (DF): Instituto para o Desenvolvimento da Saúde; 2001 [acesso 2009 Dez 10]. Disponível em: www.ids-saude.org.br/enfermagem
8. Lupton D. Risk. Nova York (US): Routlege; 1999.

9. Colomé JS, Oliveira DLLC. A educação em saúde na perspectiva de graduandos de Enfermagem. Rev Gaúcha Enferm. 2008 Set 29(3):347-53.

10. Backes DS, Erdmann AL, Büscher A. O cuidado de enfermagem como prática empreendedora: oportunidades e possibilidades. Acta Paul Enferm 2010; 23(3):341-7.

11. Colomé JS. A formação de educadores em saúde na graduação em enfermagem: concepções dos graduandos [dissertação]. Porto Alegre (RS): Escola de Enfermagem, Universidade Federal do Rio Grande do Sul; 2007.

12. Minayo MCS. O desafio do conhecimento: pesquisa qualitativa em saúde. $11^{\text {a }}$ ed. São Paulo: HucitecAbrasco; 2008.

13. Cervera DPP, Parreira BDM, Goulart BF. Educação em saúde: percepção dos enfermeiros da atenção básica em Uberaba (MG). Cien Saude Colet. 2011; 16(Supl1):1547-54.

14. Valla V, Stotz EN. Participação popular, educação e saúde: teoria e prática. Rio de Janeiro: RelumeDumará; 1997.

15. Alves VS. Um modelo de educação em saúde para o Programa Saúde da Família: pela integralidade da atenção e reorientação do modelo assistencial. Interface - Comunic, Saúde, Educ. 2005 Fev; 9(16):3952.

16. Freire P. Pedagogia do oprimido. $36^{\mathrm{a}}$ ed. Rio de Janeiro: Paz e Terra; 2003.

17. Costa RKS, Miranda FANM. Formação profissional no SUS: oportunidades de mudanças na perspectiva da Estratégia de Saúde da Família. Trab Educ Saúde. 2009 Fev; 6(3):503-17.

18. Wendhausen A, Saupe R. Concepções de educação em saúde e a estratégia de saúde da família. Texto Contexto Enferm. 2003 Jan-Mar; 12(1):17-25.

19. Saupe R. Competência dos profissionais de saúde para o trabalho interdisciplinar. Interface - Comunic, Saúde, Educ. 2005 Set-Dez; 9(18):521-36.

20. Villa EA, Aranha AVS. A formação dos profissionais da saúde e a pedagogia inscrita no trabalho do programa de saúde da família. Texto Contexto Enferm. 2009 Out-Dez; 18(4):680-7. 